\title{
Variáveis maternas e infantis associadas à ocorrência de anemia em crianças nos serviços de atenção básica em São Paulo
}

\author{
Mother and child factors related to occurrence of anemia in children assisted at primary health care \\ centers of São Paulo city, Brazil
}

Patrícia Colombo Compri' ${ }^{1}$ Maria Cristina F. S. Cury², Neil Ferreira Novo ${ }^{3}$, Yára Juliano ${ }^{3}$, Dirce Maria Sigulem ${ }^{4}$

RESUMO

Objetivo: Analisar variáveis maternas e infantis associadas à ocorrência de anemia em crianças atendidas por serviços de atenção básica à saúde do município de São Paulo.

Métodos: Participaram do estudo 357 crianças, de quatro a 24 meses, de três serviços de atenção básica à saúde da região sul da cidade. As variáveis foram separadas em categorias relacionadas à mãe e à criança. A dosagem de hemoglobina foi realizada por punção digital, com ponto de corte de $11 \mathrm{~g} / \mathrm{dL}$ para o diagnóstico de anemia. O teste $t$ de Student foi aplicado para comparar as médias de hemoglobina de dois grupos independentes; a ANOVA, para três ou mais grupos; e o teste de Mann-Whitney para comparar a velocidade de crescimento e o grupo etário.

Resultados: A prevalência de hemoglobina abaixo de $11 \mathrm{~g} / \mathrm{dL}$ foi de $60 \%$. Entre as variáveis maternas, não se constatou associação com a ocorrência de anemia. Houve associação entre anemia, gênero da criança e velocidade de ganho de peso. Quanto à alimentação, não foi encontrada associação entre tempo de aleitamento materno e anemia, mas houve associação com a ingestão quantitativa e qualitativamente pobre em ferro.

Conclusões: A alta prevalência de anemia relacionou-se ao gênero masculino, no qual a velocidade de crescimento em menores de seis meses e naqueles com 18 a 24 meses foi maior, além de se associar à baixa ingestão de ferro na dieta.

Palavras-chave: anemia; ferro; dieta; serviços de saúde; criança.

\section{ABSTRACT}

Objective: To analyze mother and child factors associated with the occurrence of anemia in children assisted at primary health care centers in São Paulo city, Brazil.

Methods: The study enrolled 357 children from four to 24 months assisted at three health care centers in the southern region of the city. Possible factors associated to anemia and related to mother and children characteristics were categorized. Hemoglobin was determined by digital puncture and the cut value for anemia diagnosis was $11 \mathrm{~g} / \mathrm{dL}$. Student t test was used to compare the hemoglobin means of two independent groups; ANOVA for three or more independent groups, and Mann-Whitney was used to test the association of anemia and age group or speed of growing.

Results: The prevalence of anemia was $60 \%$. Anemia was not associated to maternal variables. Weight gain and male gender were associated to a higher prevalence of anemia. Anemia was also associated with poor qualitative or quantitative ingestion of iron. The occurrence of anemia was not avoided by breastfeeding practice.

Conclusions: The high prevalence of anemia was related to masculine gender, which had a faster speed of weight gain in children less than six months old and from 18 to 24 months old. Anemia was also related to low ingestion of iron in the diet.

Key-words: anemia; iron; diet; health services; child. 1Professora titular da Disciplina de Saúde Coletiva da Faculdade de Medi-
cina da Universidade de Santo Amaro (Unisa), São Paulo, SP, Brasil
${ }^{2}$ Diretora da Faculdade de Medicina da Unisa, São Paulo, SP, Brasil
${ }^{3}$ Professor da Pós-Graduação em Saúde Materno Infantil da Faculdade de
Medicina da Unisa, São Paulo, SP, Brasil
${ }^{5}$ Coordenadora do Programa de Pós-Graduação em Saúde Materno Infantil
da Unisa, São Paulo, SP, Brasil
Endereço para correspondência:

Patrícia Colombo Compri

Rua Borba Gato, 331, apto.14, bloco F - Alto da Boa Vista

CEP 04747-030 - São Paulo/SP

E-mail: colombo@greco.com.br

Recebido em: 29/5/2007

Aprovado em: 11/10/2007 


\section{Introdução}

O combate à anemia carencial ferropriva, considerada um dos principais problemas da área da Saúde Pública em todo o mundo, atualmente é uma das prioridades dos profissionais da área de saúde e nutrição ${ }^{(1)}$.

A anemia ferropriva não é um problema de Saúde Pública restrito a países em desenvolvimento; contudo, é importante considerar que as condições favoráveis para o agravamento da carência de ferro estão atreladas às condições sociais e econômicas das classes de renda mais baixa, seja por uma alimentação quantitativa e qualitativamente inadequada, seja pela precariedade de saneamento ambiental ou por outros indicadores que direta ou indiretamente possam contribuir para sua elevada prevalência ${ }^{(2)}$.

Apesar da ausência de um levantamento nacional, existe consenso na comunidade científica de que a anemia ferropriva tem alta prevalência em todo território nacional, atingindo todas as classes de renda ${ }^{(3)}$. No entanto, por meio de estudos pontuais em diversas regiões país, observa-se, nas últimas décadas, um aumento significativo da prevalência e gravidade de anemia ferropriva nos grupos de risco, independentemente do nível socioeconômico ${ }^{(4,5)}$. Na faixa etária de menores de dois anos, a proporção de anêmicos se situa entre 50 e $83 \%{ }^{(6)}$.

Sabe-se que a anemia interfere nos processos de crescimento e desenvolvimento da criança, levando a algumas consequiências que devem ser salientadas como prejuízo no desenvolvimento mental, motor e da linguagem, alterações comportamentais e psicológicas como falta de atenção, fadiga e insegurança, além da diminuição da atividade física ${ }^{(7)}$.

Admite-se que a ocorrência endêmica de anemia na infância decorra da combinação entre necessidades excepcionalmente elevadas de ferro impostas pelo crescimento e dietas pobres no mineral, sobretudo ferro de alta biodisponibilidade ${ }^{(8,9)}$.Entre outros determinantes da anemia em crianças, encontram-se a prematuridade e o baixo peso ao nascer, a insuficiente ingestão de ferro em crianças com menos de 24 meses e o desmame precoce $^{(10,11)}$. O curto tempo de aleitamento materno exclusivo, a introdução tardia ou insuficiente de alimentos ricos em ferro e o consumo inadequado de estimuladores de sua absorção têm sido também apontados como causas de anemia em crianças ${ }^{(12-14)}$.

Assim como todos os problemas de Saúde Pública, a anemia ferropriva tem sua origem em um contexto mais amplo, pois é determinada não só por fatores biológicos como por condições socioeconômicas e culturais vigentes ${ }^{(15)}$. Em vista do impacto da deficiência de ferro no crescimento físico e no desenvolvimento mental, é importante que os profissionais de saúde tenham conhecimento da magnitude do problema e que existam, nos serviços, programas voltados para prevenção e vigilância da anemia e de outros eventos sanitários a ela relacionados ${ }^{(16)}$.

O presente estudo teve como objetivos analisar as variáveis maternas e infantis associadas à ocorrência da anemia ferropriva em crianças menores de 24 meses, atendidas por serviços de atenção primária à saúde da região sul do município de São Paulo.

\section{Métodos}

Estudo transversal realizado em três serviços de atenção primária: Unidade de Saúde da Família (USF) de Jordanópolis, Unidade Básica de Saúde (UBS) de São Norberto e Centro de Saúde Escola (CSE) Dr. Ananias Pereira Porto, parceiros da Faculdade de Medicina da Universidade de Santo Amaro (Unisa) na região sul de São Paulo, ligados ao Sistema Único de Saúde (SUS).

Este trabalho foi aprovado pelo Comitê de Ética em Pesquisa da Universidade Federal de São Paulo e foi solicitada a assinatura do termo de consentimento livre e esclarecido pela mãe ou responsável pela criança, antes da sua inclusão no estudo.

A seleção das crianças foi realizada nos próprios serviços de saúde, por escolha aleatória da demanda, no período de 2000 a 2003. Foram incluídas 357 crianças de quatro a 24 meses, que constituíam a demanda dos serviços de saúde no período, distribuídas da seguinte forma: 173 na UBS; 120 no USF e 64 no CSE. Foram excluídas crianças menores de quatro e maiores de 24 meses e/ou portadoras de doenças crônicas que poderiam interferir nos resultados do exame.

Para determinar o nível de hemoglobina e o diagnóstico de anemia, todas as crianças foram submetidas a exame de dosagem de hemoglobina por punção digital e leitura pelo método colorimétrico da cianometahemoglobina, com o aparelho portátil Hemocue ${ }^{\circledR}$.

Como ponto de corte e diagnóstico da anemia foi adotado o critério da World Health Organization (WHO) $)^{(17)}$, que considera anemia em crianças entre seis meses e seis anos quando os valores de hemoglobina são inferiores a $11 \mathrm{~g} / \mathrm{dL}$. Considerou-se anemia de maior gravidade quando a taxa de hemoglobina foi inferior a $9,5 \mathrm{~g} / \mathrm{dL}^{(18)}$.

As crianças que participaram do estudo foram acompanhadas e tratadas nos seus respectivos serviços de saúde: as não anêmicas receberam dose profilática de ferro e as anêmicas, dose terapêutica ${ }^{(19)}$.

As variáveis estudadas foram separadas em três categorias: relacionadas à mãe (escolaridade, idade e realização de pré- 
natal); relacionadas à criança (gênero, idade, peso ao nascer e velocidade de ganho de peso) e relacionadas à alimentação (aleitamento materno exclusivo em meses, quantidade em $\mathrm{mg}$ e densidade em $\mathrm{mg} / 1.000 \mathrm{kcal}$ de ferro da dieta).

$\mathrm{O}$ peso ao nascer foi classificado segundo os critérios estabelecidos pela WHO, considerando-se baixo peso a criança com $<2.500 \mathrm{~g}$ e peso adequado quando $\geq 2.500 \mathrm{~g}^{(20)}$. O aleitamento materno predominante foi considerado quando a criança foi alimentada ao seio e recebeu, além do leite materno, água ou bebidas à base de água, como sucos de frutas e chás ${ }^{(21)}$. A velocidade de ganho de peso das crianças foi calculada a partir do delta percentual, no qual o peso atual foi subtraído do peso ao nascer e dividido pelo peso atual multiplicado por 100 .

Para avaliar o consumo médio de ferro pela dieta da criança, foi realizado o inquérito recordatório de 24 horas, relativo ao consumo alimentar do dia anterior ao da entrevista, em uma amostra aleatória das crianças estudadas $(n=55)$. O consumo de nutrientes de ferro biodisponível foi calculado com o programa computadorizado de composição alimentar NUT (Sistema de Apoio à Nutrição, versão 2.5) ${ }^{(22)}$. O consumo de 24 horas de ferro foi expresso em termos absolutos (mg de ferro/dia), considerando-se a dose recomendada entre seis e 59 meses de idade de $10 \mathrm{mg} / \mathrm{dia}^{(23)}$.

$\mathrm{Na}$ avaliação do consumo alimentar de menores de 24 meses, considerou-se a densidade de ferro, além do consumo de energia e o consumo de ferro da dieta. A densidade de ferro da dieta relaciona o consumo absoluto de ferro ao consumo absoluto de energia, sendo expressa em mg de ferro por $1.000 \mathrm{kcal}$. Embora não haja recomendações específicas para a densidade de ferro na dieta, considerando-se a necessidade energética média na faixa etária de seis a 59 meses de idade em cerca de $1.300 \mathrm{kcal} /$ dia e a recomendação única quanto ao consumo de ferro $(10 \mathrm{mg} / \mathrm{dia})$, chega-se a uma densidade desejável de $8,0 \mathrm{mg} / 1.000 \mathrm{kcal}^{(9)}$.

Para analisar os resultados, utilizou-se o teste $t$ para comparar as médias de hemoglobina de dois grupos independentes e a ANOVA para três ou mais grupos, além do teste de Mann Whitney para comparar a velocidade de crescimento e o grupo etário. O qui-quadrado foi aplicado para diferença entre proporções de anêmicos e não anêmicos. Fixou-se o nível de significância em 5\%(24).

\section{Resultados}

Das 357 crianças estudadas, 198 (55\%) eram do gênero masculino e a média de idade, 12,2 meses. A prevalência de anemia nas crianças estudadas foi $60 \%$.

Dentre as variáveis relacionadas à mãe da criança, não se encontrou associação estatística entre anemia e os fatores de risco estudados.

Na Tabela 1, apresentam-se os níveis médios de hemoglobina e respectivos desvios padrão, mostrando haver diferença estatística entre os gêneros e não ocorrendo o mesmo com o

Tabela 1 - Nível médio de hemoglobina (g/dL), desvio padrão (DP) e valores mínimo e máximo segundo gênero, peso ao nascer, idade e tempo de aleitamento materno

\begin{tabular}{|c|c|c|c|c|}
\hline & Média \pm DP & Mín-Máx & $\mathbf{N}$ & Valor de $p$ \\
\hline Gênero & & & & 0,01 \\
\hline Masculino & $10,3 \pm 1,5$ & - & 198 & \\
\hline Feminino & $10,7 \pm 1,4$ & - & 159 & \\
\hline Peso ao nascer (g) & & & & $>0,05$ \\
\hline$<2500$ & $10,1 \pm 1,7$ & - & 43 & \\
\hline$\geq 2500$ & $10,5 \pm 1,4$ & - & 135 & \\
\hline Idade (meses) & & & & $>0,05$ \\
\hline$<6$ & $10,4 \pm 1,3$ & $7,6-13,8$ & 91 & \\
\hline $6-12$ & $10,4 \pm 1,5$ & $6,4-14,3$ & 94 & \\
\hline $12,1-18$ & $10,6 \pm 1,6$ & $6,5-14,7$ & 80 & \\
\hline$>18$ & $10,5 \pm 1,5$ & $7,0-13,7$ & 92 & \\
\hline Total & $10,5 \pm 1,5$ & $6,4-14,7$ & 357 & \\
\hline Aleitamento (meses) & & & & $>0,05$ \\
\hline$<3$ & $10,5 \pm 1,6$ & $6,4-14,3$ & 122 & \\
\hline $3-5$ & $10,5 \pm 1,4$ & $7,2-14,7$ & 156 & \\
\hline$\geq 6$ & $10,5 \pm 1,4$ & $7,3-13,8$ & 55 & \\
\hline Total & $10,5 \pm 1,5$ & $6,4-14,7$ & 333 & \\
\hline
\end{tabular}


peso ao nascer. No gênero masculino, a prevalência de anemia foi $64 \%$ e, no feminino, $55 \%$. Dentre as crianças com baixo peso ao nascer, $33 \%$ apresentavam hemoglobina menor do que $11 \mathrm{~g} / \mathrm{dL}$, comparadas a $22 \%$ daquelas com peso igual ou acima de $2.500 \mathrm{~g}$.

$\mathrm{Na}$ Tabela 1, apresentam-se os níveis médios de hemoglobina e respectivos desvios padrão, discriminados segundo a idade em meses e o tempo de aleitamento materno, não havendo diferença estatística. A prevalência de anemia nos quatro grupos etários estudados foi: $68 \%$ nos menores de seis meses; $54 \%$ nas crianças de seis a 12 meses; $54 \%$ nas crianças de 12 a 18 meses; e $64 \%$ nas de 18 a 24 meses. Quanto ao tempo de aleitamento materno exclusivo, observou-se que, nas crianças com menos de seis meses de aleitamento exclusivo, a prevalência de anemia foi de $60 \%$, enquanto que naquelas com tempo maior ou igual a seis meses foi $54 \%$.

Dentre as variáveis relacionadas à criança, o sexo masculino apresentou maior velocidade de crescimento antes dos seis meses e dos 18 a 24 meses de idade (Gráfico 1).

A ingestão de ferro foi de $4,7 \mathrm{mg} /$ dia e a densidade de ferro da dieta de $5 \mathrm{mg}$ de ferro $/ 1.000 \mathrm{kcal}$, mostrando inadequação de cerca de 50\% em relação à recomendação.

\section{Discussão}

Na primeira avaliação, $60 \%$ das crianças apresentaram níveis de hemoglobina abaixo do ponto de corte que discrimina a presença de anemia. Embora haja recomendação para a suplementação de ferro em lactentes pela Sociedade Brasileira de Pediatria ${ }^{(17)}$, a alta prevalência observada decorre da não utilização de profilaxia medicamentosa com

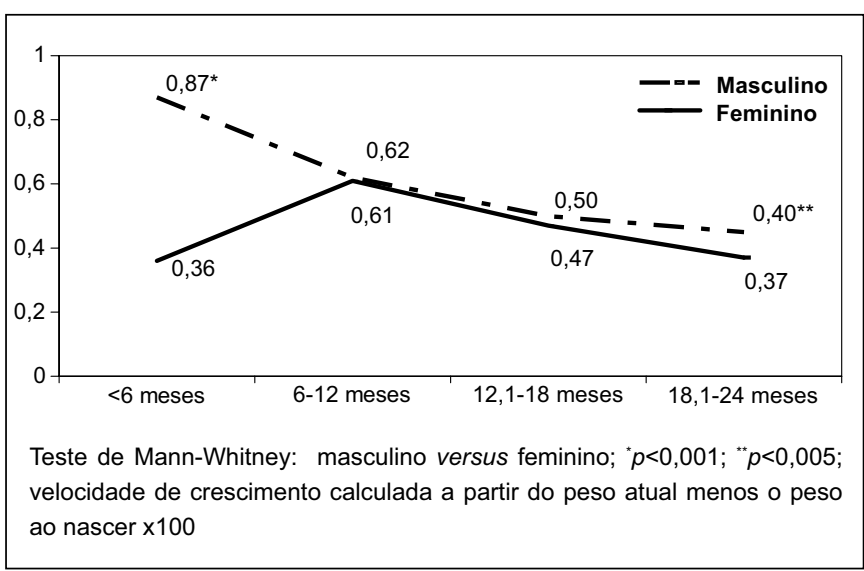

Gráfico 1 - Velocidade de crescimento por grupo etário das crianças estudadas, segundo gênero sais de ferro no período anterior à coleta de dados nas crianças estudadas. Vale ressaltar que a prevalência da anemia encontrada na população estudada está em concordância com dados de outros estudos, nos quais a mesma se situa entre 50 e $83 \%{ }^{(25)}$.

A escolaridade dos pais pode ser considerada um fator socioeconômico importante na determinação da anemia, tendo em vista que a maior escolaridade repercute em maior chance de emprego e, conseqüentemente, de renda, o que, por sua vez, condiciona melhor acesso aos alimentos ${ }^{(2)}$. No presente estudo, esta associação não foi encontrada, fato que já havia sido observado por outros autores em crianças menores de 24 meses $^{(4)}$.

A assistência ao pré-natal a ao parto devem ser eficientes no sentido de evitar e corrigir os principais problemas relacionados à saúde e à nutrição da gestante, os quais podem desencadear baixo peso ao nascer e prematuridade, considerados fatores de risco para anemia. Gestantes adolescentes apresentam maior risco de ter filhos prematuros e com baixo peso ao nascer, os quais apresentam menores reservas de ferro e, por conseguinte, maior predisposição à anemia. Além disso, tais mães são mais inexperientes quanto aos cuidados com a criança ${ }^{(2,13)}$. Apesar destas considerações, não foi constatada associação estatística entre anemia e realização de pré-natal no presente estudo, uma vez que não houve diferença na frequiência ao pré-natal entre as mães de crianças anêmicas e não anêmicas.

A maioria dos autores demonstra que, em crianças, não existe diferença entre gêneros na prevalência de anemia ou do nível de concentração de hemoglobina. Torres $e t{ }^{(6)}$, por outro lado, mostram que a prevalência da anemia pode variar entre os gêneros e afirmam que a maior prevalência da anemia no sexo masculino poderia ser explicada pela maior velocidade de crescimento apresentada pelos meninos, acarretando maior necessidade de ferro ao organismo, o que não seria suprido pela dieta ${ }^{(26)}$. Neste estudo, encontrou-se diferença estatística entre gênero e nível médio de hemoglobina, sendo este nível mais elevado no gênero feminino. Tal achado poderia ser explicado pela maior velocidade de crescimento apresentada no gênero masculino.

Recém-nascidos de baixo peso, por desnutrição intrauterina ou prematuridade, acumulam menor quantidade de ferro em comparação com os nascidos a termo, pois o mais importante armazenamento deste mineral ocorre no último trimestre de vida intra-uterina ${ }^{(27)}$. $\mathrm{O}$ baixo peso ao nascer é um dos fatores predisponentes mais importantes 
na gênese de anemia por carência de ferro. Também foi estudado o peso ao nascer das crianças, pois é sabido que este pode resultar em aumento do risco de anemia nos dois primeiros anos de vida devido à incorporação de ferro pelo feto ser proporcional ao seu peso corporal ${ }^{(10,27,28)}$. Entre as crianças de baixo peso, foi observado nível médio de hemoglobina de $10,1 \mathrm{~g} / \mathrm{dL}$, enquanto naquelas de peso normal, a hemoglobina média foi $10,5 \mathrm{~g} / \mathrm{dL}$, sem diferença estatística. Apesar deste resultado, o peso ao nascer parece ser possível fator de risco para anemia, visto que 33\% das crianças nascidas com baixo peso eram anêmicas de maior gravidade $(\mathrm{Hb}<9,5 \mathrm{~g} / \mathrm{dL})$, enquanto que, entre as de peso normal, este percentual foi de $22 \%$.

Os estudos nos quais as crianças são analisadas por grupos etários são unânimes em demonstrar prevalências mais acentuadas em crianças de seis a 24 meses. Nos primeiros dois anos de idade, o crescimento e o desenvolvimento da criança encontram-se em aceleração e as necessidades de ferro são aumentadas ${ }^{(2,29)}$. Assim, neste estudo, a faixa etária mostrou associação significante com a anemia. $\mathrm{O}$ aumento da freqüência de anemia nos primeiros anos de vida, com pico entre 12 e 24 meses e sua posterior diminuição, também foi apontado em outros estudos ${ }^{(30)}$. A prevalência de anemia em crianças de seis a 12 meses (54\%) e de 12 a 18 meses (54\%) não apresentou diferença estatística. Quando estes valores são comparados aos de crianças com menos de seis meses (68\%) e de 18 a 24 meses (64\%), há diferença estatística. Explicam-se tais achados pela maior velocidade de crescimento encontrada nestes dois extremos de grupos etários estudados. Outros fatores que podem explicar o fenômeno são a alta prevalência de desmame precoce, o atraso na introdução de alimentos ricos em ferro na dieta da criança e a maior prevalência de doenças como diarréia e infecções respiratórias nos primeiros anos de vida ${ }^{(10)}$.

$\mathrm{O}$ aleitamento materno nos primeiros seis meses de vida é fator de proteção para anemia, principalmente se exclusivo. Encontra-se menor prevalência de anemia em crianças amamentadas ao seio por um período igual ou superior a seis meses ${ }^{(10)}$. Após seis meses de vida, o ferro da alimentação torna-se indispensável para suprir a necessidade do nutriente ${ }^{(14)}$. No presente trabalho, não se detectou presença e tempo de aleitamento materno predominante como fatores de proteção para a anemia. Entre as crianças amamentadas ao seio, o nível médio de hemoglobina foi $10,5 \mathrm{~g} / \mathrm{dL}$ e, para as não amamentadas, $10,1 \mathrm{~g} / \mathrm{dL}$. A prevalência da anemia em crianças em aleitamento materno igual ou superior a seis meses foi $54 \%$ e, para aquelas com em aleitamento materno exclusivo por menos de seis meses, a prevalência foi $60 \%$, não havendo diferença estatística.

Entre os fatores que lideram as causas da anemia ferropriva, possivelmente a dieta inadequada em ferro e, especialmente a sua baixa biodisponibilidade, seja o mais importante. As reservas de ferro do nascimento aos seis meses de idade, quando em aleitamento materno, atendem às necessidades fisiológicas da criança, sem que esta necessite de complementação. A biodisponibilidade do ferro, entretanto, pode diminuir em $80 \%$ quando outros alimentos são introduzidos. Portanto, a introdução precoce de alimentos complementares é considerada como fator de risco para o aparecimento de anemia ferropriva ${ }^{(2,30)}$.

No Brasil, um estudo no município de São Paulo revelou que $49 \%$ das crianças avaliadas tinham dietas deficientes em ferro, com valores muito abaixo dos recomendados ${ }^{(31)}$. Outro estudo indicou a necessidade de suplementação profilática de ferro durante o primeiro ano de vida, uma vez que $44 \%$ das crianças avaliadas ingeriam dietas com quantidades de ferro abaixo do recomendado ${ }^{(32)}$. Também no município de São Paulo, Sichieri et al ${ }^{(27)}$ observaram o consumo de $6,2 \mathrm{mg}$ de ferro por dia, enquanto, na cidade do Rio de Janeiro, Lacerda e Cunha ${ }^{(30)}$ notaram consumo de $7,3 \mathrm{mg}$ de ferro por dia, sendo ambos os estudos realizados em crianças com faixa etária entre 12 e 24 meses.

Sigulem et al ${ }^{(4)}$, estudando crianças de seis a 60 meses no município de São Paulo, encontraram inadequação de consumo de ferro em $93 \%$ das crianças de seis a 24 meses. O consumo médio de ferro encontrado no presente estudo foi muito aquém da recomendação da Food and Agriculture Organization e da WHO $(10 \mathrm{mg} / \mathrm{dia})^{(23)}$, pois a ingestão média do mineral ferro foi de $4,76 \mathrm{mg}$, ou seja, $50 \%$ de inadequação quanto à recomendação.

Com base na importância da alimentação de desmame para assegurar o atendimento das necessidades nutricionais de ferro no primeiro ano de vida, analisou-se o consumo de alimentos de alta biodisponibilidade e estimuladores da absorção de ferro. Ao avaliar as dietas de crianças em relação à qualidade e quantidade de ferro ingerido, observou-se haver maior ingestão de ferro de origem vegetal do que animal, com maior inadequação do consumo de ferro total nos menores de 24 meses $^{(31)}$. Sendo assim, o estudo dos fatores específicos da dieta relacionados à absorção de ferro são de extrema importância para compreender o quadro epidemiológico do problema ${ }^{(32)}$. Os inquéritos nutricionais apresentam variabilidade individual e em grupos, 
a qual deve ser considerada na análise dos resultados. No entanto, acredita-se que os resultados encontrados para o grupo etário de menores de 24 meses sejam amostra do que ocorre na população geral desta faixa etária.

A variável infantil associada à ocorrência de anemia foi o gênero masculino. A velocidade de crescimento nas idades inferiores a seis meses e de 18 a 24 meses foi maior no gêne- ro masculino, reafirmando a necessidade de suplementação férrica. A implementação de ações educativas em relação aos alimentos ricos em ferro e sua biodisponibilidade, o aprimoramento do conhecimento dos profissionais de saúde acerca do problema e a necessidade de um seguimento adequado da administração de ferro são indispensáveis para o combate e o controle da carência.

\section{Referências bibliográficas}

1. Hadler MC, Juliano Y, Sigulem DM. Anemia do lactente: etiologia e prevalência. J Pediatr (Rio J) 2002;78:321-6.

2. Osório MM. Fatores determinantes da anemia em crianças. J Pediatr (Rio J) 2002;78:269-78.

3. Brasil - Ministério da Saúde. Compromisso social para redução da anemia por carência de ferro no Brasil. Brasília: Ministério da Saúde, 1999.

4. Sigulem DM, Tudisco ES, Athaide MM, Vaisman E. Anemia ferropriva em crianças no município de São Paulo. Rev Saude Publica 1978;12:168-78.

5. Szarfarc SC, Stefanini MLR, Lerner BR. Anemia nutricional no Brasil. Cad Nutr 1995;9:5-24.

6. Torres MA, Sato K, Queiroz SS. Anemia em crianças menores de dois anos atendidas nas unidades básicas de saúde no Estado de São Paulo, Brasil. Rev Saude Publica 1994;28:290-4.

7. Walter T, de Andraca I, Chadud P, Perales CG. Iron deficiency anemia: adverse effects on infant psychomotor development. Pediatrics 1989;84:7-17.

8. Hadler MC, Colugnati FA, Sigulem DM. Risks of anemia in infants according to dietary iron density and weight gain rate. Prev Med 2004;39:713-21.

9. Monteiro CA, Szarfarc SC, Mondini L. Tendência secular da anemia na infância na cidade de São Paulo (1984-1996). Rev Saude Publica 2000;34:62-72.

10. Silva LS, Giugliani ER, Aerts DR. Prevalência e determinantes de anemia em crianças de Porto Alegre, RS, Brasil. Rev Saude Publica 2001;35:66-73.

11. Brunken GS, Silva SM, França GV, Escuder MM, Venâncio SI. Risk factors for early interruption of exclusive breastfeeding and late introduction of complementary foods among infants in midwestern Brazil. J Pediatr (Rio J). 2006;82:445-51.

12. Silva DG, Franceschini SC, Priore SE, Ribeiro SM, Szarfarc SC, Souza SB et al. Anemia ferropriva em crianças de 6 a 12 meses atendidas na rede pública de saúde do município de Viçosa, Minas Gerais. Rev Nutr 2002;15:301-8.

13. Neuman NA, Tanaka OY, Szarfarc SC, Guimarães PR, Victora CG. Prevalência e fatores de risco para anemia no Sul do Brasil. Rev Saude Publica 2000;34:56-63.

14. Souza SB, Szarfarc SC, Souza JM. Anemia no primeiro ano de vida em relação ao aleitamento materno. Rev Saude Publica 1997;31:15-20.

15. United Nations Children's Fund; United Nations University/ World Health Organization/Micronutrients Initiative. Preventing iron deficiency in women and children -technical workshop. Boston: International Nutrition Foundation; 1999.

16. Rodrigues CR, Motta SS, Cordeiro AA, Lacerda EM, Reichenheim ME. Prevalência da anemia ferropriva e marcadores de risco associados em crianças entre 12 e 18 meses de idade atendidas nos ambulatórios do Instituto de Puericultura e Pediatria Martagão Gesteira. J Pediatr (Rio J) 1997;73:189-94.
17. World Health Organization. Iron deficiency anaemia: assessment prevention and control: a guide for programme managers. Geneve: WHO; 2001.

18. DeMayer EM. Preventing and controlling iron deficiency anemia through primary health care: a guide for health administrators and programmer managers. Geneve: WHO; 1989.

19. SBP; Departamento Científico de Nutrologia da Sociedade Brasileira de Pediatria [homepage on the Internet]. Anemia carencial ferropriva [cited 2007 Sep 28]. Available from: http://www.sbp.com.br/img/documentos/doc_anemia_carencial_ferropriva.pdf

20. Puffer RR, Serrano CV. Características del peso ao nascer - publicacion científica $n .^{\circ} 54$. Washington: OMS/OPAS, 1988.

21. Brasil - Ministério da Saúde - Secretaria de Políticas de Saúde. Guia alimentar para crianças menores de 2 anos. Brasília: Ministério da Saúde, 2002.

22. Anção MS, Cuppari L, Tudisco ES, Draibe SA, Sigulem D. Programa de apoio à nutrição. $2^{a}$ ed. São Paulo: Centro de Informática em Saúde da Escola Paulista de Medicina; 1995.

23. Food and Agriculture Organization; Organización Mundial de la Salud. Necessidades de vitamina A, hierro, folato y vitamina B12: informe de uma consulta mixta de expertos. Roma: FAO; 1991.

24. Siegel S, Castellan NJ. Nonparametric statistics for the behavioral sciences. $2^{\text {nd }}$ ed. New York: McGraw-Hill; 1988.

25. Devincenzi MU, Ribeiro LC, Sigulem DM. Suplementação de ferro na infância. Diag Trat 1999;4:49-52.

26. Sachdev H, Gera T, Nestel P. Effect of iron supplementationm on physical growth in children: systematic review of randomised controlled trials. Public Health Nutr 2006;9:904-20.

27. Sichieri R, Szarfarc SC, Monteiro CA. Relação entre dieta e ocorrência de anemia ferropriva em crianças. J Pediatr (Rio J) 1998;64:169-74.

28. Bhandari N, Bahl R, Taneja S. Effect of micronutrient supplementation on linear growth of children. Brit J Nutr. 2001;85 (Suppl 2):S131-4.

29. Miranda AS, Franceschini SC, Priore SE, Euclydes MP, Araujo RM, Ribeiro $\mathrm{SM}$ et al. Anemia ferropriva e estado nutricional de crianças com idade de 12 a 60 meses do município de Viçosa, MG. Rev Nutr 2003;16:163-9.

30. Lacerda E, Cunha AJ. Anemia ferropriva e alimentação no segundo ano de vida no Rio de Janeiro, Brasil. Rev Panam Salud Publica 2001;9:294-301.

31. Szarfarc SC, Monteiro CA, Meyer M. Estudo das condições de saúde das crianças no Município de São Paulo, SP (Brasil), 1984/1985 X consumo alimentar. Rev Saude Publica 1988;22:266-72.

32. lannotti LL, Tielsch JM, Black MM. Iron supplementation in early childhood: health benefits and risks. Am J Clin Nutr 2006;84:1261-76. 